De plus, ee même procédé offre sur les procédés usuels de pasteurisation deux autres avantages non moins intéressants, sinon plus, surtout le premier des deux qui concerne l'alimentation des enfants en bas âge : $1^{\circ}$ celui de conserver presque intégralement l'acide carbonique du lait, ee qui signifie qu'il garde à l'état soluble et par conséquence parfaitement assimilable les sels de chaux et les phosphates du lait : encore un carac.ère qui le rapproche du lait cru; $2^{\circ}$ et celui d'épargner la perte en 'rolume du lait, par évaporation, qui se chiffre, dans les procédés usuels de pasteurisalion, de $11 / 2 \grave{a} 2 \%$, e'est-à-dire à plus de 1 à 2 centimes par litre, au prix actuel du lait.

\title{
LA SOLUBILISATION DE LA MATIÈRE ORGANIQUE DU LAIT. SES APPLICATIONS
}

\author{
par R. VLADESCO \\ Professeur de Chimie biologique à la Faculté de Médecine Vétérinaire de Bucarest.
}

\section{DOSAGE DE LA GRAISSE}

A l'oceasion des nombreuses destructions de matière organique entreprises dans le laboratoire de ehimie biologique de M. Gabriel Bertrand, à l'Institut Pasteur, j'ai fait la remarque que voici :

Lorsqu'on laisse refroidir le liquide clair résulté de la solubilisation des tissus animaux traités par le mélange azoto-sulfurique, avant qu'il noircisse, on constate que la graisse se sépare sous la forme d'une pellicule qui nage à la surface du liquide.

Ce fait d'observation a été le point de départ de nombreux essais faits ultérieurement dans le but d'instituer une méthode pour doser la graisse des tissus animaux.

Pour des raisons de commodité, j'ai commencé mes recherches avec le lait. Depuis le commencement j'ai dû remplacer le mélange azoto-sulfurique par l'acide azotique seul, pour éviter la précipitation de sels de calcium et par conséquent pour faciliter la séparation de la graisse.

Après plusieurs tâtonnements, je me suis arrêté à la technique suivante :

Le lait, additionné d'une quantité déterminée d'acide azotique concentré, est chauffé jusqu'à ce que le liquide devienne tout à fait transparent. On laisse refroidir ensuite et on sépare la graisse par. filtration sur un papier filtre ordinaire.

A priori il était à prévoir que la quantité de graisse obtenue devait ètre dépendante : 
10 De la quantité d'acide azotique ajouté au lait :

$2^{\circ}$ De la température à laquelle on chauffe ;

$3^{0}$ De la durée du chauffage.

L'expérience a confirmé amplement ces prévisions. Voici quelques expériences à l'appui :

$1010 \mathrm{cc}$. lait, $10 \mathrm{cc}$. acide azotique concentré, chauffés quinze minutes sur un bain de sable : 0,566 gr. de graisse.

$10 \mathrm{cc}$. lait, $20 \mathrm{cc}$. acide azotique concentré, chauffés quinze minutes toujours sur un bain de sable : $0,542 \mathrm{gr}$. de graisse ;

$2^{\circ} 10 \mathrm{cc}$. lait, $10 \mathrm{cc}$. acide azotique chauffés quinze minutes sur une plaque d'éternit : $0,576 \mathrm{gr}$. de graisse.

10 ec. lait, $10 \mathrm{cc}$. acide azotique, chauffés trente minutes sur une plaque d'éternit : 0,420 gr. de graisse.

Mais lorsqu'on travaille dans les mêmes conditions, on trouve les mêmes résultats. Voici un exemple :

$20 \mathrm{cc}$. lait, $20 \mathrm{cc}$. d'acide azotique, chauffés pendant une heure au bain-marie : 0,709 gr. de graisse.

10 ce. lait, 10 ec. d'acide azotique, chauffés pendant une heure au bain-marie : $0,357 \mathrm{gr}$. de graisse.

5 cc. lait, 5 cc. d'acide azotique, chauftés pendant une heure au bain-marie : $0,177 \mathrm{gr}$. de graisse.

La conclusion qu'on en peut tirer, c'est qu'il est possible d'utiliser l'observation ci-dessus mentionnée pour le dosage des matières grasses du lait à condition d'employer toujours la même technique.

Il est à peine besoin de faire remarquer que tout ce qu'on peut prétendre d'une telle méthode, c'est de donner les richesses relatives en graisses des laits analysés et pas plus.

A ce point de vue elle présente le même degré d'exactitude que la méthode Gerber, sans avoir ses avantages au point de vue pratique, au moins sous la forme sous laquelle je l'ai utilisée.

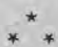

Dans mes recherches systématiques, je n'ai pas poussé plus loin que l'éclaircissement complet du mélange : lait+acide azotique, la destruction de la matière organique.

Pour ce faire, on chauffe directement sur la flamme d'un brûleur Teclu, $10 \mathrm{cc}$. de lait additionné de 20 ec. d'acide azotique concentré dans un ballon KJELDAнL de 100 cc. en agitant continuellement pour éviter les soubresauts et la projection du liquide en dehors.

L'opération dure environ cinq minutes.

$\mathrm{Au}$ liquide encore chaud, on ajoute $10 \mathrm{cc}$. d'eau et on le refroidit en plongeant le ballon KJeLdahL dans un bassin rempli d'eau froide.

Afin d'éviter la prise en masse de la graisse séparée, on secoue 
vigoureusement le ballon plongé dans l'eau froide pendant le refroidissement. Cette dernière opération peut être effectuée sous le jet d'un robinet à eau. Lorsque la graisse s'est complètement solidifiée, ce qu'on observe facilement, on filtre le liquide à travers un papierfiltre préalablement taré dans une fiole à bouchon rodé.

Pourvu que le ballon KJeldahl ait été bien nettoyé avant la destruction et qu'on ait suivi les prescriptions ci-dessus indiquées, toutes les particules de graisse peúvent être facilement amenées sur le filtre par quelques lavages à l'eau froide.

Le filtre contenant la graisse est ensuite introduit dans la fiole à l'émeri et desséché dans une étuve à air see.

Après refroidissement dans la eloche à dessécher, on pèse de nouveau. La différence entre les deux pesées donne la quantité de graisse contenue dans les $10 \mathrm{ce}$. de lait.

\section{$* *$}

Dans le tableảu qui suit, sont exposées les analyses faites sur 30 échantillons de lait fourni par les laitiers ambulants de Bucarest :

\begin{tabular}{|c|c|c|c|c|c|}
\hline $\begin{array}{c}\text { Echantillon } \\
\text { analysé }\end{array}$ & $\begin{array}{l}\text { Graisse } \\
\text { trouvée } \\
0 / 0\end{array}$ & $\begin{array}{l}\text { Echantillon } \\
\text { analysé }\end{array}$ & $\begin{array}{l}\text { Graisse } \\
\text { trouvée } \\
00\end{array}$ & $\begin{array}{l}\text { Echantillor } \\
\text { anaiysé }\end{array}$ & $\begin{array}{c}\text { Graisse } \\
\text { trouvée } \\
0 / 0\end{array}$ \\
\hline M. T. . & $1,61 \mathrm{gr}$. & V. G. . & $2,72 \mathrm{gr}$. & I. A. . & $4,01 \mathrm{gr}$. \\
\hline A. C. . & $1,98-$ & I. T. . & 2,88 - & I. L. . & $4,75-$ \\
\hline I. C. . . & $2,06-$ & M. I. . . & $2,96-$ & I. C. : & $4,78-$ \\
\hline T. B. . . & $2,10-$ & P. N... & $3,20-$ & G. R. . & $5,05 \div$ \\
\hline I. S. . . & $2,24-$ & D. C. . . & $3,21-$ & I. B. . & $5,08=$ \\
\hline D. I. . . & $2,34-$ & A. C. . & $3,40-$ & T. S. . & $5,21-$ \\
\hline F. V. : & $2,33-$ & A. C. . & $3,46-$ & E. D. . & $5,34-$ \\
\hline I. M. , & $2,49-$ & I. M. . & $3,57-$ & F. V. . & 5,69 \\
\hline R. V. . & $2,66-$ & M. M. . . & $3,89-$ & M. F. . & $5,93 \div$ \\
\hline I. I. . & $2,68-$ & S. I. : . & $3,91-$ & G. P. . & $6,22-$ \\
\hline
\end{tabular}

Quoique de ce qui précède, il ressorte avec netteté qu'on ne peut pas prétendre de cette méthode des chiffres qui représentent la teneur absolue en graisse des laits analysés, il m'a semblé intéressant de. comparer les résultats obtenus à l'aide du procédé déerit avec ceux obtenus à l'aide d'une méthode rigoureuse et dont le principe soit autant que possible rapproché sinon le mème.

J'ai choisi la méthode de Kumagawa-Suto pour la raison que ce sont les acides gras qui sont dosés par elle et non les glycérides neutres.

Les recherches faites dans cette direction ont abouti toutes à la conclusion suivante :

En suivant la technique ci-dessus exposée, on trouve toujours dans le lait une quantité de graisse sensiblement plus grande qu'avec la méthode de Kumagawa-Suto.

Pour avoir une idée de cet écart, je cite trois analyses prises au hasard parmi mes expériences. 
Quantité de lait analysé.

$10 \mathrm{ce}$.

$10-$

$10-$
Graisse trouvée par la méthode de Kumagawa-Sute.

$0,467 \mathrm{gr}$.

$0,288-$

0,166

Graisse trouvée après la solubilisation azotique.
$0,563 \mathrm{gr}$.
0,357 -
$0.195-$

La mème observation, exactement, est à faire lorsqu'on fait la comparaison avec la méthode de Gerber.

En voici quelques exemples :

$\begin{array}{ccc}\text { Laits analysés } & \begin{array}{c}\text { Graisse trouvée par la } \\ \text { méthode de Gerber. }\end{array} & \begin{array}{c}\text { Graisse trouvée } \\ \text { solubilisation az }\end{array} \\ \text { No 1. . . . . . . } & 5,2 & 5,59 \\ \text { No 2. } & 5,5 & 5,87 \\ \text { No 3. . } & 5,6 & 5,93 \\ & *_{*}^{*} & \end{array}$

A quoi tiennent ces différences ?

C'est pour résoudre cette question que j'ai fait quelques expérienees sur les acides gras dont je disposais dans mon laboratoire : acide stéarique et acide oléique.

Ces corps furent traités de la même manière que le lait, c'est-à-dire ehauffés pendant cinq minutes avec de l'acide azotique étendu de la moitié de son volume d'eau.

Les quantités pesées du premier corps ont été toujours presque égales à celles prises en recherche.

Pour l'acide oléique, au contraire, elles ont été, sans aucune exception, sensiblement plus élevées.

Sans préjuger, quant au processus ehimique qui se passe avec ce corps, sous l'influence de l'acide azotique à chaud, je me contente, pour l'instant, de signaler ce fait comme suffisant pour expliquer les différences dont il s'agit plus haut.

Les expériences suivantes font voir la manière dont se comportent les deux acides gras à étudier lorsqu'ils sont soumis à l'action de l'acide azotique dans les conditions décrites. De même, on peut apprécier l'augmentation de poids éprouvée par l'acide oléique :

$\begin{array}{lccc} & \text { Quantité prise. } & \text { Durée de chauffage. } & \text { Quantité trouvée. } \\ \text { Acide stéarique. } & 0,926 & 7 \text { minutes } & 0,920 \\ \text { Acide oléique. . } & 1,856 & 7 & - \\ \text { Acide stéarique. . } & 1,048 & 3 & 2,059 \\ \text { Acide oléique. . . } & 1,737 & 3 & 1,040 \\ & & * & \end{array}$

Il est possible de faire de telles déterminations sur des quantités beaucoup plus réduites de lait. La seule précaution à prendre dans ce càs, e'est d'éviter l'évaporation trop rapide du mélange lait + acide azotique, en se servant d'un vase approprié. J'ai fait des analyses 
de graisse mème sur un centimètre cube de lait en utilisant des tubes à essai en verre Iéna pour solubiliser la matière organique. La technique à suivre est exactement la même que celle dans laquelle on emploje $10 \mathrm{cc}$.

Les recherches comparatives faites sur le même lait montrent que les résultats ne sont pas tout à fait concordants, même si la durée du chauffage est la même. Cela se conęoit facilement d'ailleurs. Voici deux exemples :

10 Dosage sur 10 ce de lait : $133 \mathrm{~m}$. gr. de graisse ;

Dosage sur 1 ce. du même lait : $11 \mathrm{~m}$. gr. de graisse ;

20 Dosage sur $10 \mathrm{ec}$. de lait : $98 \mathrm{~m}$. gr. de graisse;

Dosage sur 1 ec. du même lait : $8 \mathrm{~m}$. gr. de graisse.

Quoiqu'il en soit, je erois que le méthode exposée dans cette note, par sa simplicité et par le fait d'être appliquable à des quantités de lait très réduites, peut rendre des services dans nombre de circonstances où il s'agit de recherches comparatives.

\title{
LE PROCÈS DE LA MATIËRE GRASSE DU LAIT
}

\author{
par Ch. PORCHER
}

- suite -

Le mélange des laits de plusieurs animaux. Ses diverses modalités. - Si nous abandonnons le lait de mélange des diverses traites d'un seul animal, lequel n'a d'intérêt pratique que dans certaines cireonstances dont il a été question, pour envisager maintenant le lait de mélange de plusieurs animaux, bien des cas peuvent se présenter. L'amortissement des oscillations que je signalais tout à l'heure lorsqu'il s'agissait du mélange des laits d'un même animal, va également s'observer ici, et le calcul des probabilités nous indique déjà que plus il y aura d'animaux, plus il y aura de chances pour que ces amortissements soient mieux effectués. Les laits de mélanges ont des physionomies très pariées. Rassembler le lait de deux animaux différents, c'est déjà faire un mélange. Rassembler le lait d'un grand nombre d'animaux, comme le font les Sociétés de ramassage qui approvisionnent les grandes villes, e'est aussi faire un mélange; mais il y a de nombreux intermédiaires entre ces deux cas, et c'est d'eux que je voudrais discuter maintenant.

Dans un lait individuel, on a une grande variable : la matière grasse. Dans un lait de mélange, la richesse butyreuse de l'échantillon moyen peut se traduire par la formule : $\Sigma \frac{M G \text {. }}{n}, n$ étant le nombre d'animaux dont les laits constituent le mélange. La question 